\title{
Introduction to high-resolution cryo-electron microscopy
}

\begin{abstract}
$\mathbf{F}_{\mathrm{p}}^{\mathrm{o}}$

or many years two techniques have dominated structural biology - X-ray crystallography and NMR spectroscopy. Traditional cryo-electron microscopy of biological macromolecules produced macromolecular reconstructions at resolution limited to 6-10 Å. Recent development of transmission electron microscopes, in particular the development of direct electron detectors, and continuous improvements in the available software, have led to the "resolution revolution" in cryo-EM. It is now possible to routinely obtain near-atomic-resolution 3D maps of intact biological macromolecules as small as $\sim 100 \mathrm{kDa}$. Thus, cryo-EM is now becoming the method of choice for structural analysis of many complex assemblies that are unsuitable for structure determination by other methods.
\end{abstract}

\section{INTRODUCTION}

Structural biology strives to understand the processes of life at the level of single molecules and atoms. This is achieved by determining the positions of each of the thousands of atoms that comprise biological macromolecules with great precision. But how does one describe the structure of molecules that are too small to be seen even by the best optical microscope?

The first and by far most successful method that allows the determination of the exact position of each atom in a molecule is X-ray crystallography [1]. This powerful technique has dominated the field of structural biology for the last 60 years and is responsible for almost $90 \%$ of the structures that have been deposited in the Protein Data Bank (PDB), the main database of structures of all biomolecules [2]. In crystallography, the structure of the analysed macromolecule is calculated from the diffraction pattern that is recorded after illumination of the crystals of this molecule with a bright beam of X-rays. The incident electromagnetic wave is scattered by the array of scatterers (i.e., single atoms or, more precisely, their electrons). If the atoms are arranged periodically (i.e., in a crystal), then the light that is scattered by them also interferes periodically, ultimately leading to a diffraction pattern (i.e., a series of discrete spots where all of the scattered light interferes constructively). The location and intensity of these spots depend on the structure of the macromolecular crystal. The wavelength of the incident light should be on the same order of magnitude as the spacing between lattice planes in the crystal. Such a wavelength (expressed in angstroms $[\AA]$ ) corresponds to X-rays. Knowing the exact wavelength and other parameters of the experimental setup, one can calculate the electron density of the molecules that form the analysed crystal and predict the exact location of each atom. One parameter is the phase of light in each reflection point, which cannot be recorded directly and must be calculated separately, most frequently based on additional diffraction experiments or using homologous structures. The most significant bottleneck for X-ray crystallography is the necessity to obtain well-diffracting crystals of the sample of interest. The range of the diffraction area determines the maximal resolution of the obtained structure. In high-resolution structures $(<2.0 \AA)$, most of the atoms can be placed unambiguously in the obtained electron densities. Moderate resolution (2.5-3.5 $\mathrm{A}$ ) allows one to trace the backbone of the peptide main chain and positions of most of the side chains. Low resolution structures $(>4 \AA)$ can only provide information on the secondary structures or overall shape of the molecule. For many proteins, including membrane proteins and dynamic and complex molecular assemblies, obtaining diffracting crystals may be very difficult or even impossible.

Another technique that allows the determination of the atomic structures of proteins and other biomolecules is nuclear magnetic resonance (NMR) spectroscopy [3]. Nuclear magnetic resonance spectroscopy is responsible for nearly $10 \%$ of all of the structures that are deposited in the PDB. In this technique, a

\section{Mariusz Czarnocki-Cieciura}

\section{Marcin Nowotny}

Laboratory of Protein Structure, Internationa Institute of Molecular and Cell Biology, $4 \mathrm{Ks}$. Trojdena St., 02-109 Warsaw, Poland

Laboratory of Protein Structure, Internationa Institute of Molecular and Cell Biology, Warsaw, Poland, e-mail: mnowotny@iimcb. gov.pl

Received: June 2, 2016

Accepted: June 27, 2016

Key words: electron microscopy, cryo-EM, negative stain, single particle reconstruction, structural biology

Abbreviations: cryo-EM - cryo-electron microscopy; CTF - contrast transfer function; EM - electron microscopy; TEM - transmission electron microscopy; SPR - single particle reconstruction

Acknowledgements: We thank prof. dr hab. $n$. med. Romuald Wojnicz and dr n. med. Edyta Reichman-Warmusz at the Department of Histology, School of Medicine with the Division of Dentistry, Medical University of Silesia in Katowice, Zabrze, Poland for providing the TEM micrographs presented in figure 3 . 
concentrated sample in aqueous solution is subjected to a strong magnetic field. At specific (resonance) frequencies, nuclei that have an intrinsic magnetic moment (i.e., non-zero spin) can absorb and re-emit electromagnetic radiation. These frequencies depend mainly on the composition of the nucleus and its chemical surrounding (i.e., covalent bonds and atoms that are located in the closest proximity), which introduce small changes in the recorded frequencies, called chemical shifts. After assigning recorded resonance frequencies to individual atoms in the analysed sample, this information can then be used to model its local structure. Nuclear magnetic resonance spectroscopy is the method of choice for relatively small molecules $(<20-30 \mathrm{kDa})$. Larger molecules have a slower tumbling rate, which causes faster relaxation times and leads to broadening and decay of the NMR signal. Additionally, resolving and assigning several hundreds or thousands of recorded resonances becomes challenging. Sample preparation for NMR is much easier, because crystallization is not required. Finally, NMR spectroscopy can also be used to study the dynamics of the macromolecules in solution and can provide structural insights into intrinsically unstructured proteins.

Electron microscopy (EM) has long remained a low-resolution technique that provides only information on the overall shape of macromolecules [4]. This information has often been combined with high-resolution crystal structures of subunits or subcomplexes of the larger assemblies that are studied by EM. The advantage of EM is that it allows direct visualization of the analysed molecules, provided they are sufficiently large. The major difficulty associated with this technique is the very weak contrast of biological macromolecules in a hydrated state, which sets the lower size limit for the analysed particles to approximately $100 \mathrm{kDa}$. Proteins and other biomolecules can be visualised by transmission electron microscopy (TEM) if they absorb electrons differently than the surrounding material. For this purpose, two alternative techniques were developed. In negative stain EM, the sample is immobilized on the surface of a carbon film and covered with a thin layer of stain that strongly absorbs electrons. The analysed molecules are then visible as bright holes in a uniform layer of stain. In cryo-EM, the sample is embedded in a thin layer of vitrified water. This allows direct visualization of the analysed molecules without any artefacts that are introduced by staining, but it does not provide high contrast between the sample and surrounding water. An analysis of individual particles that are visualised by EM can only provide information on the overall shape of the molecule in a specific projection. Single particle reconstruction (SPR) allows the analysis of many thousands of such pictures and can model the three-dimensional (3D) envelope (i.e., 3D EM map - map of the Coulomb potential, in contrast to the electron density maps generated in X-ray crystallography) that would produce similar projections. In negative staining EM, after the analysis of many thousands of particles, the resolution of the obtained model can typically achieve 20-30 , whereas the traditional cryo-EM technique can be used to reconstruct models of large and symmetrical virus particles up to 6-10 A. Recent advances in EM improved this limit in resolution. Many cryo-EM structures can now achieve near-atomic resolution (up to $\sim 2 \AA$ ) [5]. With EM maps of such quality, it is now often possible to build the atomic model of a macromolecular complex residue-by-residue de novo, without any prior knowledge of its structure [6]. This places EM among the other high-resolution techniques: X-ray crystallography and NMR spectroscopy. In fact, EM is now becoming the method of choice for the structural analysis of many complex assemblies that are unsuitable for structure determination by other methods.

\section{HISTORY}

The first optical microscopes that were constructed beginning in the late $16^{\text {th }}$ century were usually equipped with only single lenses. Around 1675, Antony Van Leeuwenhoek developed the methodology of creating superior glass lenses, and he is believed to have built the first true microscope with magnification close to $300 \times$ [7]. During the next two centuries, people learned how to combine different lenses to reduce chromatic and spherical aberrations and increase the final magnification, but the magnification could not be increased infinitely. In the 1870s, Ernst Abbe formulated and experimentally tested a mathematical theory that connected the resolution limit of an optical microscope $(d)$ with the wavelength of light $(L)$ and numerical aperture $(N A)$ of the microscope: $d=L /(2 \times N A)$.

The numerical aperture can also be defined as $n \times \sin (\theta)$, where $n$ is the index of refraction of the medium in which the lens is working, and $\theta$ is the maximal half-angle of the cone of light that can enter or exit the lens. For modern lenses, $N A$ can reach a value of approximately 1.4. Thus, optical microscopes that operate at wavelengths of visible light (400-700 $\mathrm{nm}$ ) can be used to observe objects that are larger than $\sim 200$ $\mathrm{nm}$. However, this range is not very useful in structural biology because most proteins and protein complexes are only a few nanometres in diameter. Thus, it became clear that it is necessary to use much shorter wavelengths to visualise biomolecules. This led to the development of soft X-ray microscopes. However, because of the specific properties of $X$ rays (e.g., they are difficult to produce and focus and easily penetrate most biological samples), the resolution of such microscopes is practically limited to $\sim 20 \mathrm{~nm}$.

The solution to this problem arose with the use of particles other than photons. Since late $19^{\text {th }}$ century, people learned how to produce cathode rays (i.e., streams of particles that are emitted by a negative electrode in a vacuum tube). In 1897, J.J. Thomson demonstrated that these rays are composed of very light, negatively charged particles that were later called electrons. The real breakthrough came in 1926, when Hans Busch showed that the electron beam can be focused by a short solenoid similarly to the way glass optics focus visible light. Electrons that are accelerated by high voltage also have a very short wavelength. According to the de Broglie hypothesis that was formulated in 1924, these and all other particles can be described as waves with a wavelength $(L)$ that is related to their momentum $(p)$ through the Planck constant $(h): L=h / p$.

For electrons that are accelerated by a voltage of $300 \mathrm{kV}$ (which is typical for modern top-end cryo-EM microscopes), this gives a wavelength of $2 \mathrm{pm}$ (by taking into account relativistic effects), which is five orders of magnitude shorter 
than for visible light. Electrons thus appeared as ideal particles for constructing super-high-resolution microscopes. This idea came to fruition in 1931 when Ernst Ruska and Max Knoll built the first proof-of-principle electron microscope [8]. Two years later, the first working electron microscope was built by Ruska, which was capable of resolving down to $50 \mathrm{~nm}$ and significantly better than any visible light microscope. During the next decades, the resolution of electron microscopes gradually increased, mainly because of improvements in the lenses, electron sources, and application of higher accelerating voltages. Modern electron microscopes typically operate at a few hundred to a few thousand kilovolts and can achieve a resolution of $\sim 0.1 \mathrm{~nm}$, which is practically limited not by Abbe's equation but rather by the quality of the lens. Despite the great progress that has been made over the last 70 years, electron microscopes are still based on the same concept as Ruska's prototype.

The subnanometre resolution of electron microscopes allows the direct visualisation of proteins and other large biomolecules. The development of such microscopes, however, did not initially revolutionize the field of structural biology. The main reason for this is that biological samples (or hydrated materials in general) have very low optical contrast and usually need special preparation and staining techniques to become visible by electron microscopes (see Section: "Sample Preparation"). The energy of electrons that are used for imaging is sufficiently high to cause severe radiation damage to delicate samples by breaking the atomic bonds and causing secondary damage that is caused by the radicals that are generated. The first problem can be solved by the negative staining technique [9]. Because the stain is usually quite stable, it also reduces the problem of radiation damage. The major drawback of this method is that it limits the level of detail that is visible on the recorded image. Moreover, staining can influence the state or shape of the analysed molecules, and such bias is very hard to control. An alternative solution, developed by Jacques Dubochet's group in 1984 [10], is to stabilize and protect the sample by embedding it in a thin, vitrified layer of water. This technique, called cryo-electron microscopy (cryo-EM), allows the recording of much more detailed pictures of biological samples than negative staining, but it is limited by the very weak contrast (i.e., the density of the sample relative to its surroundings) [11]. The contrast can be increased using longer exposure times, but that can destroy the vitrified sample. Thus, to increase the signal-to-noise ratio, averaging thousands of aligned molecules is typically necessary. Because of the very low signal-to-noise ratio, this process cannot be easily automated and can only be done for homogeneous samples.

In the single particle reconstruction (SPR) procedure, images of the analysed molecules that are viewed from different angles and recorded using either the negative stain or cryo-EM technique, can be used for the reconstruction of their 3D envelope. The resolution of such a model was limited mainly by the number and quality of the recorded images and typically reached a value of 10-20 А. Recent ad ances in hardware and software allowed the recording and analysis of high-resolution images of biological macromolecules. The first structures with near-atomic resolution were reported for large, helical viruses, for which image processing was much easier because of their high level of symmetry $[12,13]$. The true breakthrough came in 2012 with the introduction of direct electron detectors (DEDs) that significantly increased the quality of images that are recorded with a low electron dose and enhanced the resolution of structures that are obtained by cryo-EM. Since that time, nearly 200 EM structures have been published with a resolution better than $4 \AA$.

To accommodate the growing number of 3D EM structures of biological macromolecules, the Electron Microscopy Data Bank (EMDB) was founded at the European Bioinformatics Institute in 2002 [14]. Five years later, the EMDataBank portal was created to unify access to this database and other cryo-EM resources [15]. Today, the total number of deposited EM maps (3370 as of May 2016) is still far less than the number of published X-ray structures (over 100,000), but the dynamic growth of the EMDataBank indicates that we are now undergoing a cryo-EM revolution. Many important examples of recently deposited, high-resolution 3D EM maps include highly symmetrical protein complexes, such as the $2.8 \AA$ structure of $20 S$ proteasome [16] and $2.3 \AA$ structure of human AAA ATPase p97 [17]. Because of continual improvements in computational algorithms, high-resolution structures can now also be obtained for molecules with low or no symmetry. Recent examples include eukaryotic and prokaryotic ribosomes that were solved to approximately $3 \AA[18,19], 2.2 \AA$ structure of $\beta$-galactosidase [20] and $1.8 \AA$ structure of glutamate dehydrogenase (currently the highest-resolution structure obtained by cryo-EM) [21]. Importantly, cryo-EM can now address many of the most important biological issues that require the determination of the structure and architecture of large, dynamic, and complex molecular assemblies that are mostly unattainable by X-ray crystallography. Cryo-EM has already been extensively used to characterize the structure and mechanism of action of many such macromolecular complexes, including the spliceosome and snRNP complexes (for a recent review, see [22]), anaphase-promoting complex [23], ion channels $[24,25]$, and human $\gamma$-secretase [26]. Cryo-EM is now at the forefront of structural biology, allowing the determination of structures that were previously not achievable.

\section{ELECTRON MICROSCOPES}

There are two types of electron microscopes: transmission electron microscopes and scanning electron microscopes. Transmission electron microscopy (TEM) is based on electrons that are transmitted through the ultra-thin sample. Because of this, it is similar to imaging in inverted optical microscopes. The images that are recorded by TEM are two-dimensional (2D) projections of the analysed samples that provide details about their internal composition. In contrast, scanning electron microscopy (SEM) produces a 3D image of the surface of the analysed sample based on electrons that are scattered from it. Samples that are used for SEM need to be electrically conductive. Thus, biological samples need special preparation. They must be fixed, dried, and coated with a thin layer of a conducting material. Because of this preparation procedure and because scanning electron microscopes generally have a much lower resolu- 

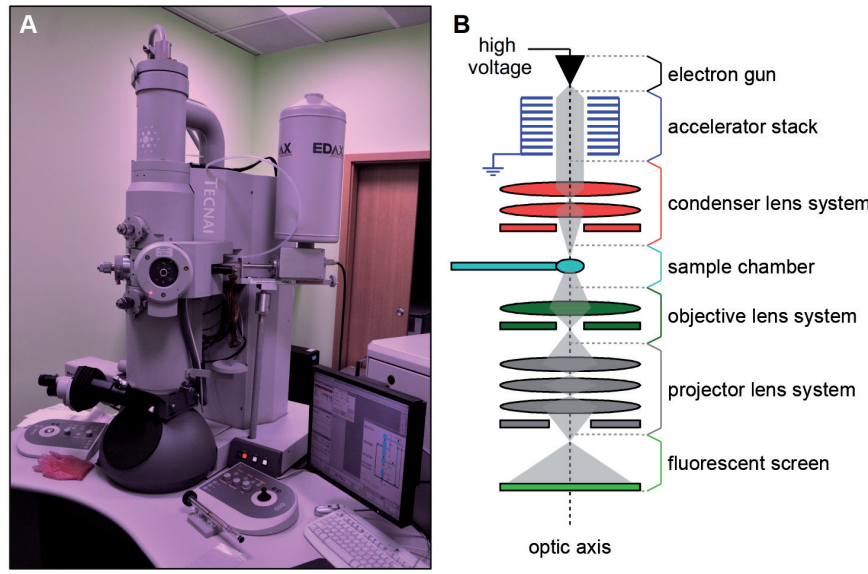

Figure 1. Transmission electron microscope and TEM grids. A. Tecnai 120 kV D1203 BioTwin microscope in the Department of Histology and Embryology, Medical University of Silesia, Zabrze, Poland. B. General layout of a transmission electron microscope. C. A picture of square mesh copper support TEM grid. D. Two types of carbon coating in TEM grids.

tion than transmission electron microscopes, only TEM is currently used in structural biology. The following sections will be dedicated solely to TEM.

Every transmission electron microscope consists of an electron column with the specimen chamber that forms the body of the microscope and several auxiliary components, including the vacuum system $[27,28]$. The electron column contains three major components: the electron source, several lenses that focus the electron beam, guide it through the sample, and produce magnification, and the imaging system on which the image of the sample is projected (Fig. 1A, $\mathrm{B})$. The high vacuum in the electron column minimizes the collision frequency of the electrons with gas atoms and ensures stable operation of the electron gun. It is created and maintained by several types of pumps (mechanical/rotary pumps, oil-diffusion pumps, turbo-molecular pumps and ion pumps) that operate within different pressure ranges.

The electron source produces a coherent electron beam. Two types of electron sources are used in electron microscopes that are based on different physical principles: thermionic electron guns and field emission guns. In thermionic electron guns, electrons are emitted from a heated filament, typically a bent tungsten (W) wire or sharp lanthanum hexaboride $\left(\mathrm{LaB}_{6}\right)$ crystal. Such electron guns are relatively inexpensive, but have relatively low brightness, and heating the filament causes broadening of the energy distribution of the emitted electrons. In field emission guns (FEGs), the electrons are emitted from a very sharp, pointed cathode by the strong electric field that is created close to its tip. Field emission guns generally produce more coherent and much brighter electron beams, but are much more expensive. In thermal/warm FEGs, field emission is assisted by thermal energy. If the gun is kept at an ultra-high vacuum $(<10-11$ Torr), then the FEG source can also operate at ambient temperature (cold FEG), which provides even better temporal coherence, but such sources suffer from rapid degradation and are very expensive. The electrons that are emitted from the electron gun are then accelerated through an accelerator stack toward the anode by the electric field that is parallel to the optical axis. This field is generated by the accelerating voltage (typically $100-300 \mathrm{kV}$ ) that is applied between the electron source and the positively charged (usually grounded) anode, and it determines the final kinetic energy of the electrons that are emitted by the electron source.

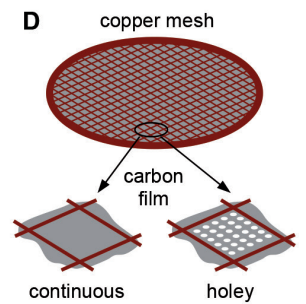

The most important component of every microscope is the lens that is responsible for creating the magnified image of the sample. Electrons that are produced by the electron gun, like all moving charged particles, are bent by the Lorentz force in the presence of electromagnetic field. In electromagnetic lenses that are used in electron microscopes, a strong magnetic field is produced by a coil that is wound into a short, tightly packed helix (solenoid) that carries a direct current. The shape of this field allows bending the electron beam similarly to the way glass lenses bend a light beam; the only difference is that the image that is produced is rotated because of the left-hand rule. The focal length of such an electromagnetic lens depends on the current that passes through the solenoid and can be easily regulated. All lenses in electron microscopes are grouped into three systems (Fig 1B): condenser lens system, objective lens system and projector lens system. Each system consists of at least four components: deflectors that regulate the exact position of the beam and are used for aligning the microscope, one or more electromagnetic lenses, a stigmator that corrects for the residual astigmatism of the lenses, and aperture that regulates the convergence angle and intensity of the beam. The condenser lens system consists of two condenser lenses and is used to direct the beam into the sample. It regulates the beam intensity, beam coherence, and size and centering of the beam spot that illuminates the sample. The objective lens system produces a magnified real image of the sample and is used for focusing. The projector lens system consists of several intermediate lenses that allow easier regulation of the magnification and the final projector lens that projects the magnified image onto a detector. The final magnification of an electron microscope is the algebraic product of the magnification factors of each of the imaging lenses and can be varied over a wide range (typically $10^{3}-10^{6}$ ).

Several types of detectors can be used in TEM [29]. Because the visualization of delicate biological samples requires a very low dose of electrons, detection of the image was the limiting factor in structural biology for many years. In TEM, images of the sample were traditionally projected onto a fluorescent screen. Images can also be recorded using photographic film, which offers very good resolution but cannot be easily automated and requires many time-consuming manipulations. The first electronic detectors that were used in EM were based on charge-coupled device (CCD) cameras and were introduced in 1980s [30]. They offer a superior dynamic range and allow the relatively easy automation of the data collection process. However, because 
of the high energy of the electrons that is used in EM, they must be coupled with an electron scintillator screen. Such an indirect electron detection system limits the resolution of the recorded image. Thus, for high-resolution imaging in cryo-EM, photographic films were still in use at the beginning of the $21^{\text {st }}$ century. The true revolution that enabled the routine collection of near-atomic-resolution images of intact biological samples came with the development of direct electron detectors (DEDs), which are based on complementary metal-oxide-semiconductor (CMOS) devices [31]. They directly convert electrons to charges, which significantly improves the resolution of the recorded image and provides better sensitivity and signal-to-noise ratio. Direct electron detectors also offer a much faster read-out, which allows the recording of many images per second, resulting in so-called movies. Such movies can then be processed to correct for beam-induced sample movement. This typically results in large amounts of data. Thus, one of the major challenges in single particle reconstruction of cryo-EM samples is the processing of large datasets that can be easily and automatically recorded with modern TEM detectors.

The last major component of an electron microscope is the sample chamber that is located in the middle of the objective lens system where the sample is inserted in a dedicated specimen holder [27]. Most TEM microscopes are equipped with a side-entry stage, where the specimen is manually inserted through an airlock that is used to preserve the high vacuum that is present in the electron column. Some modern electron microscopes that are dedicated to cryo-EM are now equipped with automatic sample loaders that minimize the user interactions with the microscope. The specimen stage allows manipulation of the sample via the dedicated specimen holder inside the electron microscope (shifting and tilting). It has to be very stable to minimize sample movements during the exposure. In cryo-EM, the specimen holder also must be cooled by liquid nitrogen to maintain a constant temperature of the vitrified sample. Thus, the specimen stage is often the most mechanically complex part of a transmission electron microscope.

\section{OVERVIEW OF THE METHOD}

Cryo-EM is becoming the routine technique for obtaining near-atomic-resolution structures of biological macromolecules. In the following sections, the main steps of sample preparation, data collection and analysis are discussed. More detailed information on the cryo-EM technique and all of the steps described below can be found in excellent reviews that were published recently [11,32-36].

\section{SAMPLE PREPARATION}

Every TEM sample must be fitted into a dedicated sample holder and is typically circular with a diameter of $3.05 \mathrm{~mm}$. Biological macromolecules are prepared on metal discs called grids [36]. Their solid support structure is made with a fine copper mesh (other metals like gold or nickel can also be used), often covered with an additional formvar layer (a type of polyvinyl formal resin). Such grids are then covered with a thin carbon film on which a solution of the analysed particles can be applied. The uniform carbon layer can be deposited by evaporating graphite and, if thin enough, is almost transparent to electrons. Because carbon is generally hydrophobic, carbon-coated grids have to be made hydrophilic prior to loading aqueous samples. This is typically achieved by the ionized gas inside a glow discharger device. Because heavy metals that are used in negative stains provide relatively high contrast, grids with a continuous carbon film can be used directly to visualize biological macromolecules in negative stain EM. In cryo-EM, however, particles are embedded in a thin layer of vitrified water with no extra stain and they produce very weak contrast. To reduce the signal from the background, such particles are exposed directly to the beam through the holes in the carbon film. Such holey carbon film can be manually produced with the aid of a formvar emulsion [37] or by semiconductor lithographic techniques (commercially available Quantifoil ${ }^{\circledR}$ or C-Flat ${ }^{\mathrm{TM}}$ grids).

One advantage of the EM analysis of biological macromolecules compared with other structural biology techniques is that relatively small amounts of the sample are needed (typically just a few microliters of particle solution, with concentrations often as low as a few tens of micrograms per millilitre). The entire procedure typically involves three major steps: biochemical purification of the sample, assessment of its quality by negative stain EM, and final preparation of cryo-EM grids.

Similar to all structural biology techniques, cryo-EM samples must be purified to almost perfect homogeneity using standard biochemical methods [36]. Although samples with some degree of heterogeneity can be analysed using a classification procedure (see Section: "Dimensional Analysis"), it significantly complicates the calculations. Thus, it is always better to minimize the heterogeneity of the sample at the stage of purification. There are two types of heterogeneity - compositional and conformational - that need to be taken into account. Compositional heterogeneity can be usually assessed by standard biochemical procedures, such as sodium dodecyl sulphate-polyacrylamide gel electrophoresis (SDS-PAGE) and size-exclusion chromatography (SEC). Native gel electrophoresis and static and dynamic light scattering techniques can also be used to assess the polydispersity of more complex samples. Heterogeneity can then often be reduced during the purification procedure by choosing proper buffering conditions and applying different chromatographic steps. For unstable protein complexes, mild chemical cross-linking with glutaraldehyde has often proven useful [38]. Mild cross-linking can also reduce the conformational heterogeneity of some complex samples, but because many protein complexes exhibit intrinsic conformational variability that is critical for their biological function, such chemical stabilization can also introduce structural bias that must be critically assessed. Conformational heterogeneity is generally much more difficult to detect, and the best way to assess the quality of the sample is to directly visualize it by EM. Thus, almost all samples for cryo-EM are first analysed by the relatively simple negative staining EM.

Negative staining protocols involve thee major steps: adsorption of the sample on a glow-discharged carbon-coated grid, blotting the excess sample and washing the grid with 
deionized water, and staining with a heavy metal solution [39]. The dried layer of stain preserves the overall structure of the sample (or more precisely, its cast that is created during dehydration), but some structural changes (e.g., collapse of the specimen) can still occur. The concentration of the sample and the exact time of adsorption have to be adjusted to ensure the proper distribution of the particles on the grid. Sample buffer composition and washing time can affect the background signal level, and the type, concentration, and time of staining determine the contrast and quality of the stain layer. There are a variety of heavy metal stains that are used for the negative staining technique, including uranyl acetate, phosphotungstic acid, sodium silicotungstate, ammonium molybdate, methylamine tungstate and methylamine vanadate. Uranyl acetate is the most common stain, because it generates the highest contrast and has a fixative effect. Generally, however, all stains have different characteristics and are usually not inert to the sample. Negatively stained biological macromolecules appear on recorded micrographs as bright spots on a dark background. During image processing, such particles can be selected, inverted, aligned, and used for SPR (see the following sections). Because the resolution of the resulting model is typically limited to $\sim 20 \AA$, negative staining is currently used mostly to assess the quality of the sample for cryo-EM. Thus, the most critical analysis is the 2D classification procedure that can be used to assess the conformational heterogeneity of the sample (see Section: "Two-dimensional Analysis"). One of the drawbacks of the negative staining technique is that many molecules tend to stick to the carbon coating in only several preferred orientations. This might affect the final $3 \mathrm{D}$ reconstruction process, because even angular coverage of the analysed particles is critical for SPR techniques. This problem can be overcome by recording additional images of tilted specimens. Generally, for heterogeneous samples, such orientation bias can also be advantageous, because it simplifies the 2D classification procedure and can be used to analyse mixtures of particles with different conformations. Based on such an analysis, it is often possible to choose the conditions in which the sample is locked into a single conformation and can be analysed by cryo-EM.

In cryo-EM, the particles are kept in a native, hydrated state by embedding them in a thin layer of vitrified water [11]. Imaging at cryo temperatures is used to reduce specimen movement and radiation damage. The cryo-EM sample preparation is a two-step process [36]. First, the solution of the analysed particles is loaded into a holey carbon film, and the excess sample is removed by blotting. Second, the sample is rapidly frozen by plunging it into a cryogen (e.g., liquid ethane or propane, which both have a greater heat capacity than liquid nitrogen). Good cryo-EM grids have a thin layer of amorphous ice that covers most of its surface. Particles are evenly distributed, and their concentration is sufficiently high to maximize the particle count per image, but not too high to minimize their overlap. Blotting is critical for the thickness of the amorphous ice layer. It has to be sufficiently thick to accommodate a uniform layer of particles in different orientations, but not too thick to support enough contrast and ensure that all particles are in the same focus (usually a few hundred to a few thousand angstroms). Blotting also removes most of the particles from the grid, af- fecting the final concentration of the sample. Thus, cryo-EM generally requires higher concentrations of the sample than negative stain EM. Sample vitrification by plunge-freezing is critical for the formation of the amorphous ice layer. If the sample is not cooled fast enough or is heated above the devitrification temperature of $-137^{\circ} \mathrm{C}$ (e.g., during transfer from the plunger to the cryo-specimen holder or to the storage container), crystalline ice can be formed that strongly diffracts electrons and degrades image quality. Sample preparation for cryo-EM (i.e., blotting and plunging) can be achieved by simple, homemade instruments but can also be automated by several commercially available plungers (e.g., Vitrobot by FEI, Cryoplunge by Gatan). Once the sample is frozen, it can be transferred to a storage box in liquid nitrogen or directly to the pre-cooled cryo-specimen holder.

\section{DATA COLLECTION}

Cryo-EM SPR methods are used to model the 3D envelope of the specimen based on the recorded TEM images. Biological samples produce very weak excess electron scattering relative to the background. Thus, SPR methods typically require averaging over many thousands of particles to boost the signal-to-noise ratio. The final resolution of the model depends on the resolution of the recorded images (limited mainly by the radiation damage) and, more importantly, on the image contrast. Contrast can be improved during data collection by increasing the beam intensity (which is usually not possible for biological macromolecules due to radiation damage), recording images at a small defocus or by using phase plates.

In TEM, the electron beam penetrates the thin sample, and the image is formed by the electrons that are scattered on the specimens' atoms [27]. Transmission electron microscopy samples produce two types of scattering contrast: thickness contrast (a thicker sample results in the scattering of more electrons) and atomic-number contrast (atoms with higher atomic number scatter more electrons). Additionally, most electron scattering mechanisms involve a phase change that can lead to phase contrast. Biological samples yield very weak scattering contrast, because they are typically very uniform in thickness and atomic number. In negative stain EM, the contrast is boosted by the heavy metal stain. In cryo-EM, however, the particles must be visualized without any stain and at very low beam brightness to minimize radiation damage [32]. Thus, phase contrast is the major source of image contrast in cryo-EM.

The phases of the scattered electrons cannot be measured directly, but the small phase change that is introduced by the scattering phenomena can lead to interference with the electrons that pass through different parts of the sample. Such interference can occur on the detector when the image is slightly defocused and the objective aperture of the microscope is large enough to enable several diffracted beams to contribute to image formation. Defocussing is the most common method of increasing the phase contrast in cryo$\mathrm{EM}$, but it also affects the resolution of the recorded images and blurs high-resolution details. Phase contrast can also be increased by phase plates without defocusing [36]. In principle, they introduce a large phase shift $(\sim \Pi / 2)$ to all scattered 
electrons without affecting the unscattered beam. Electron microscopy phase plates (e.g., Zernike phase plates and Volta potential phase plates) were introduced only recently, but the first structures that were calculated with these plates showed that they significantly improved the image contrast and reduced the number of particles that have to be averaged during SPR [40] (for review, see [35]).

To record high-resolution images, it is critical to work on a perfectly aligned microscope [41]. All major components of the microscope should be aligned on a top-down basis. Special attention should be paid to the eucentricity adjustment (which is especially important when tilting the specimen), correction of the objective lens astigmatism (by fine-tuning the power spectrum of the image), and minimization of the residual beam tilt (coma-free alignment) [42]. Typical TEM images for SPR analysis are recorded with no specimen tilt to minimize the sample thickness and maximize the signal-to-noise ratio. Recording a series of tilted images, however, can be beneficial. A tilt-pair analysis based on pairs of images recorded at two tilt angles (typically $0^{\circ}$ and $10-20^{\circ}$ ) can be used to establish the handedness of the final model and evaluate the image-processing procedures $[43,44]$. Another technique that utilizes tilted images is cryo-electron tomography. As in medical computer tomography scanning, the 3D structure of the sample is reconstructed using this method from a set of $2 \mathrm{D}$ projections recorded at predefined tilt angles (typically from $-65^{\circ}$ to $+65^{\circ}$ ) [45]. With subtomogram averaging, cryo-electron tomography can be used to determine the 3D structure of large macromolecular complexes in situ [46], albeit with a maximum resolution of $\sim 10 \AA$, which is significantly lower than with cryo-EM SRP [47].

Data collection in the EM analysis of biological macromolecules can be divided into three steps: search, focusing, and exposure [32]. Searching is done with low magnification and a wide field of view and is used to locate an area on the grid with a good distribution of sample particles and minimal stain/ice artefacts. During focusing, high magnification and a small field of view are used to set the desired focus. In cryo-EM, the images are typically recorded with a small defocus value $(0.5-4.5 \mu \mathrm{m})$ to improve the image contrast and to facilitate the subsequent image processing procedures. In particular, images recorded at a few different defocus values have to be combined to fully extract the high resolution features of the sample (contrast transfer function [CTF] correction; see Section: "Single image processing" and Fig. 2). After focusing, images can be recorded in exposure mode with the desired magnification and field of view. In cryo-EM, the images have to be recorded with a low electron dose (typically $<20$ electrons $/ \AA^{2}$ ). Thus, the exposure is always a balance between the signal-to-noise ratio and radiation damage. The dose can be slightly increased when DEDs are used in movie mode. With the high frame rates of such detectors, direct observation of radiation damage and correction of specimen movement is possible during image processing (see Section: "Single image processing"). In SPR analysis, typically several hundred images have to be recorded, and modern cryo-electron microscopes are equipped with high-throughput data collection software that automates the image recording process.

\section{DATA PROCESSING}

Unprocessed cryo-EM images yield information about the overall shape, dimensions, and compositional homogeneity of macromolecules. Image processing can provide additional information that is not readily available from the raw data. During single image processing, all of the recorded frames are first checked and corrected for artefacts and aberrations that are introduced during data collection (i.e., CTF and motion correction, and rejection of poor quality images). During 2D analysis, individual particles are then selected, aligned, and averaged to boost the signal-to-noise ratio. This step is based on the $2 \mathrm{D}$ classification procedure and can be used to assess the conformational heterogeneity of the sample. If the initial 3D model is available, heterogeneous samples can also be analysed in more detail by a 3D classification procedure. Finally, during the 3D reconstruction procedure, a spatial model of the macromolecule (3D EM density map) is calculated and refined to best fit all of the recorded 2D projections. Near-atomic-resolution models can then be used to determine the atomic structure of the macromolecule de novo. Many computer programs have been developed to perform these analyses. Most popular software packages are Spider [48], EMAN [49], Relion [50], and Frealign [51], which was used for the refinement of many important high-resolution maps. A full list of software useful for the analysis of EM data is available on a dedicated Wikibook web page [52].

Single image processing

All TEM images are inherently distorted by many aberrations that are introduced by the microscope. These perturbations can be mathematically described by a contrast transfer function (CTF) that modulates the amplitudes and phases of scattered electrons in a spatial frequency-dependent manner (Fig. 2) [53]. This function depends on the spherical aberration coefficient of the objective lens system (which is constant for certain microscopes), the wavelength of the incident electrons (defined by the accelerating voltage), and defocus value (which is specific for each image). For a perfect image, the CTF would have a constant value of +1 , meaning no modulation of phases and amplitudes (Fig. 2D). Real CTFs have a complex, oscillatory form with several zero transitions and are damping to zero at higher frequencies because of the damping envelope function (Fig. 2A, B). The first zero point of the CTF determines the point resolution of the image, whereas the fading of the signal at higher frequencies defines the information limit of the microscope (Fig. 2A, C). The oscillatory shape of the $\mathrm{CTF}$ results in selective contrast enhancement that can be seen in the power spectrum (absolute value of the 2D Fourier transform of an image; Fig. 2B, C). This power spectrum is the sum of the 2D structure factors of all particles present in the micrograph multiplied by the CTF, and the random noise. Frequencies from the original image that correspond to $\mathrm{CTF}=0$ are lost, whereas other frequencies are either modulated pos- 


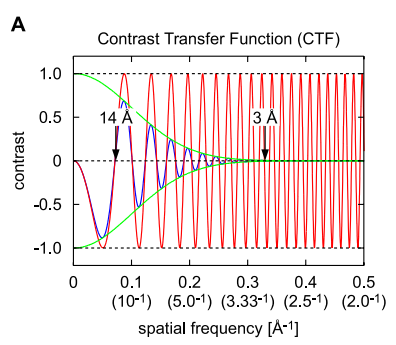

B

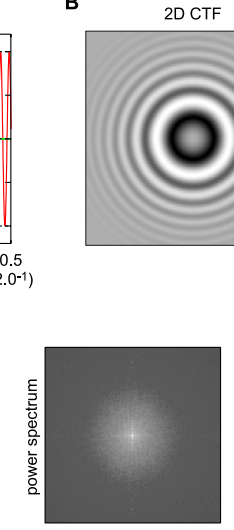

E

D
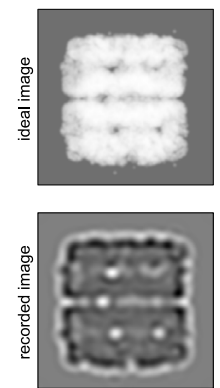

$\mathbf{F}$

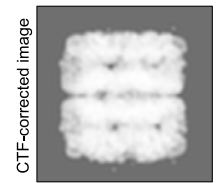

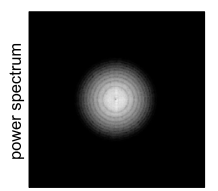

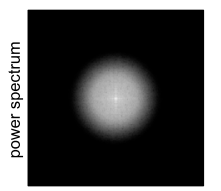

C
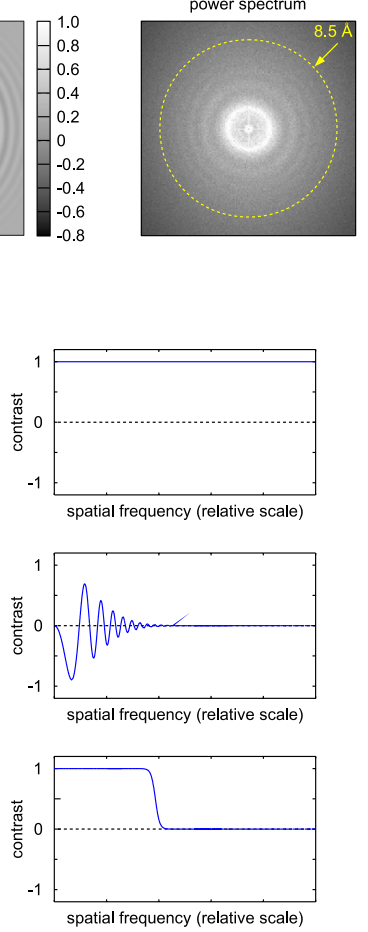

Figure 2. Contrast transfer function and CTF correction. A. The CTF (blue line) consists of two components: oscillatory function (red line) and damping envelope (green line). The first zero point of the CTF defines the point resolution, whereas damping to zero sets the information limit (14 ̊ and $3 \AA$, respectively, in this example curve). B. The same simulated CTF as a 2D function that modulates the frequencies of the image. C. Power spectrum of a real TEM image modulated by the CTF. The information limit of this image $(\sim 8.5 \AA)$ is defined by the damping oscillations. D-F. CTF correction explained on simulated data. D. Ideal image that would be recorded if the microscope did not introduce any modulations of the transmitted frequencies (theoretically ideal CTF with constant +1 value). E. Real TEM modulates the transmitted signal with the oscillatory, damping CTF, which results in a perturbed recorded image. F. During CTF correction, the 2D Fourier transform of an image is divided by the CTF that is calculated from the recorded power spectrum (E). Missing frequencies that are close to the CTF zeroes can be obtained by collecting additional images at different defocus values. This results in the restoration of all frequencies up to the information limit of the image, which limits the final resolution of the corrected image.

itively (positive CTF values) or inverted (negative CTF values). To interpret any details beyond the first $\mathrm{CTF}=0$, images have to be CTF-corrected.

During initial image processing, all recorded micrographs are usually manually reviewed. Low-quality images (e.g., those that contain high levels of crystalline ice, staining artefacts, strong spherical aberrations, or other features that might perturb the SPR procedure) are discarded, and all other are selected and processed. At this stage, CTF correction can also be applied [54]. In this procedure, the CTF curve is calculated from the power spectrum of each image and then used to correct the image for the introduced modulations (i.e., the Fourier spectrum of each image is divided by the calculated CTF; Fig. 2D-F). This restores the unperturbed information (structure factor) on all of the available frequencies, except those that are missing because of the zero values of the CTF. In the SPR procedure, however, CTF correction is typically only performed during final refinement [55]. This also allows one to obtain most of the missing frequencies by aligning images that were recorded at different defocus values [32]. If the images are recorded by a DED camera in movie mode, they can also be motion-corrected to compensate for specimen drift and beam-induced movement [11]. All of these corrections are critical for the analysis of high-resolution cryo-EM images, in which most of the information is encoded in the phase contrast, but they can also improve the quality of the 3D models obtained from negative stain data.

\section{Two-dimensional analysis}

The general principle of the SPR analysis is to calculate the 3D shape of a macromolecule based on its 2D projections [11]. To create an unbiased model, it is important to collect such projections from as many angles as possible. This can be challenging for some samples - in negative stain EM many molecules tend to adsorb to the grid only in preferred orientations, while in cryo-EM some proteins may align at the water-air interface. Because the signal-to-noise ratio of TEM images of biological samples is very low, it is necessary to increase their contrast by averaging thousands of such projections. Single-particle images have to be selected from the recorded images (i.e., particle picking) and classified according to their similarity (2D clustering). Images of similar views can then be aligned and averaged (Fig. 3). Such averaged projections have a much higher signal-to-noise ratio and can be used to calculate the initial 3D model.

During particle picking, individual projections of the macromolecule are selected from the recorded TEM images (Fig. 3A). For high-resolution SPR analysis, typically tens of thousands of such particle images have to be collected and aligned, making it often the most labour-intensive step in SPR. It is also one of the most critical steps to ensure the quality of the final 3D model. Because of the low signal-to-noise ratio of cryo-EM images, some "bad particles" (e.g., ice contaminations, different types of macromolecules, or other artefacts) cannot be easily filtered out during the subsequent classification procedure and will thus affect the 3D reconstruction. Generally, particles can be selected manually or automatically. The automated procedures are typically based on machine-learning algorithms and require several representative particles to be selected manually (semi-automated or supervised learning), but several unsupervised algorithms are also available [56].

The next step in 2D analysis is the classification of all of the selected particles according to their Euler angles. This $2 \mathrm{D}$ clustering is an important step prior to $3 \mathrm{D}$ reconstruction and can also directly answer some biological questions. Most of the available 2D classification procedures are based on the $k$-means clustering algorithm [11]. Typically, all particles are iteratively aligned to a defined number $(k)$ of templates that can be initially selected from the particle set itself (unsupervised classification) or computed from the known 3D structure of similar molecules (supervised classification). The alignment is performed using a correlation measure between each particle and the templates. In each iteration, the next generation of templates is calculated by 

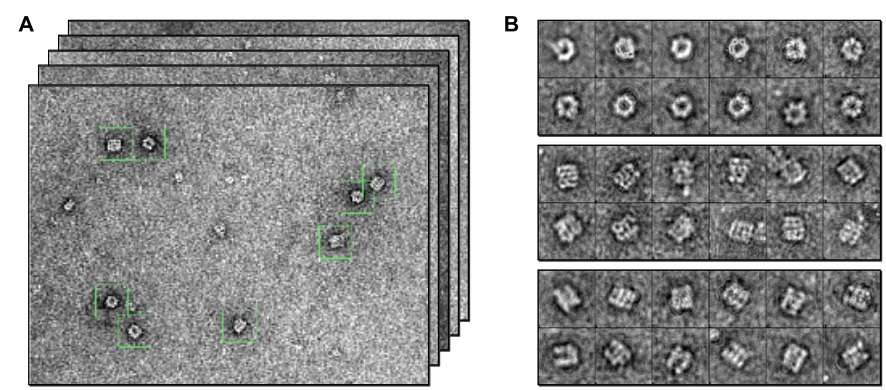

Figure 3. Image processing and class averaging during the SPR procedure. A. TEM micrographs with negatively stained GroEL complexes (Reichman-Warmusz E, Wojnicz R, Czarnocki-Cieciura M, Nowotny M, unpublished data). B, C. An example of the results of the 2D clustering procedure (B) and the corresponding class averages $(\mathrm{C})$ calculated by the EMAN2 software package [49]. Note that in unsupervised classification some class averages might represent the same projection of the macromolecule.

averaging all of the clustered particles, followed by the next round of assignment. The recent application of maximum likelihood methods to the 2D classification problem has led to significant improvements in this important step in SPR analysis [51,57].

The 2D classification procedure results in the assignment of all particles to different 2D projections of the macromolecule (Fig. 3B). All particles that are grouped in such classes can then be aligned and averaged to create class averages (Fig. 3C). Some "bad particles" that were typically present in the original particle set can be grouped together during 2D clustering and easily eliminated. Others are usually randomly distributed throughout all of the 2D classes and can contribute significantly to the overall noise. All of the calculated class averages can be used to assess the heterogeneity of the sample. Selected high-quality class averages are then used for $a b$ initio 3D structure determination [11].

Three-dimensional analysis

To reconstruct the 3D shape of a macromolecule based on 2D images, it is necessary to calculate the Euler angles of all of the projections. This is typically not a problem in cryo-electron tomography, in which the sample is rotated during data acquisition, and the angular differences between different projections are known a priori. In classic cryo-EM or negative stain SPR analysis, the Euler angles of all the particle images have to be determined computationally. In this case, the $3 \mathrm{D}$ reconstruction begins by calculating the initial 3D model [11]. This model can then be used to map all of the recorded projections and calculate their Euler angles in an iterative refinement process. All of the selected particles (including some "bad particles") contribute to the final 3D map, which has to be carefully verified to exclude any model bias and calculate the final resolution.

Selection of the initial 3D model is critical for the success of 3D reconstruction. The initial model can be generated from the existing structures or calculated ab initio directly from the class averages. In many cryo-EM analyses, the initial 3D model is generated from the low-resolution negative stain EM structure [32]. All other known high- or low-resolution structures of the sample or structurally similar mac-

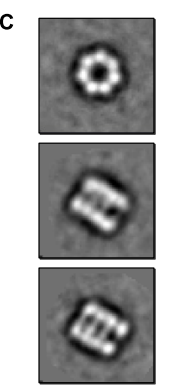

romolecules (e.g., homologous protein complexes) may also be used, but all such models must be used with caution, because any model bias introduced at this stage can influence the final 3D reconstruction. If no structural information about the sample is available, then the initial 3D model needs to be calculated $a b$ initio. This can be performed using the $2 \mathrm{D}$ Fourier transforms of the calculated class averages (unsupervised angular reconstruction; Fig. 4A). According to the central section theorem, all such transforms (2D discrete Fourier transforms calculated on the array of pixels representing each image) are central sections of the 3D Fourier transform of the analysed macromolecule (i.e., 3D structure factor). They can be used to reconstruct the initial 3D model in reciprocal space that is related to the 3D model in real space by inverse 3D Fourier transform. To this end, Euler angles of all class averages have to be predicted, and this can be facilitated by collecting additional tilted images [11]. Two of the most popular tilt procedures rely on additional pairs of images that are collected at $0^{\circ}$ and $50^{\circ}$ tilts (random conical tilt) or $-45^{\circ}$ and $+45^{\circ}$ tilts (orthogonal tilt reconstruction) [58], but an unbiased model can also be obtained directly by cryo-electron tomography [46]. Finally, any initial 3D model can be validated by the tilt-pair analysis [44].

For heterogeneous samples, 2D classification often results in grouping together different conformational states of the macromolecule or complexes with different stoichiometry. This results in biased class averages and can degrade the resolution of the final 3D EM map or even lead to false 3D models. To better characterise all particles in heterogeneous samples, 3D multireference alignment can be applied [11]. In this procedure, the assignment of all particles to different $2 \mathrm{D}$ projections of the macromolecule is aided by the 3D model. If models of different conformational states of the macromolecule are available, they can be used for supervised 3D classification; otherwise unsupervised 3D classification procedures based on the initial 3D model have to be employed $[59,60]$. This allows for dissecting different conformational states of the sample and subsequent reconstruction of the 3D models based only on a subset of all particles (see [61] for a recent example).

Model refinement is often the most computationally complex and time-consuming step in SPR analysis [11]. During this process, the initial 3D model is refined by iterative optimization of the rotational and translational parameters (Euler angles, rotation, and centering) of all of the selected (unaveraged) particles to better match the reconstruction. At the end of each round of refinement, a new 3D map is calculated. To this end, the structure of the molecule is remodelled in reciprocal space by inserting the Fourier transforms of all particles into the reconstructed volume in the optimized positions. An alternative refinement method that is based on the maximum likelihood approach was also recently introduced [62]. This statistical approach is generally more computationally complex but can often produce significantly better structures [34]. To monitor the refinement process 


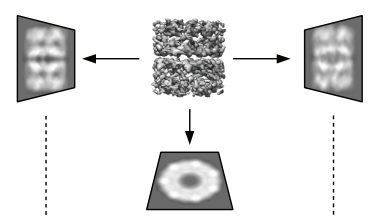

calculated 2D FT

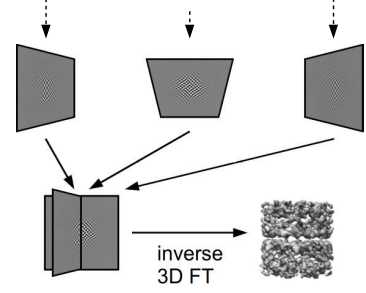

B

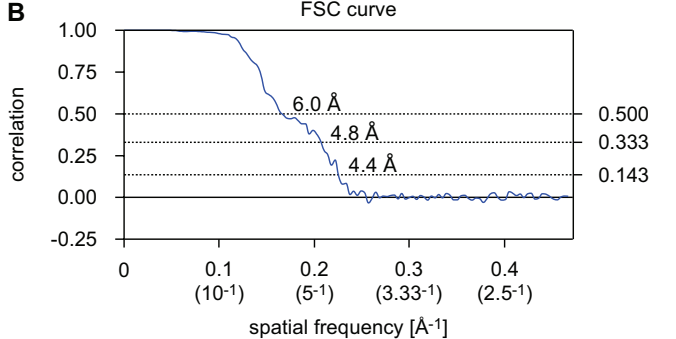

C

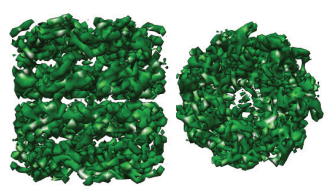

EMD-6422

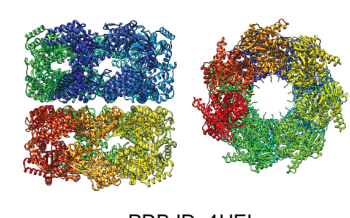

PDB ID: 4HEL

Figure 4. Explanation of the SPR 3-dimensional analysis: 3D reconstruction and structure validation. A. Central section theorem and calculation of the 3D model. The 2D Fourier transforms (FT) of all of the 2D projections (or class averages) are central sections of the 3D FT of the macromolecule. After calculation of this 3D FT model, inverse Fourier transform is used to calculate the real space 3D model. B. During refinement, two independent models are calculated from two halves of the original particle set. From their comparison, the Fourier shell correlation (FSC) curve is calculated, which relates the similarity between the two models to the spatial frequencies. The resolution of the model can be defined as the frequency at which the FSC curve drops below a certain threshold. C. Structure validation showing a $4.1 \AA$ cryo-EM map of GroEL chaperonin (EMDB-6422; Roh SH, Hryc C and Chiu W, unpublished data) compared with the $3.2 \AA$ crystallographic structure of the same molecule (PDB ID: 4HEL; Saxena AK and Meena SR, unpublished data).

and assess the quality and resolution of the final model, the original particle set is typically split into two halves, and the refinement is performed for each half independently [63]. Two resulting 3D reconstructions are then compared in Fourier space by the Fourier shell correlation (FSC) curve that describes the cross-correlation coefficient between the two structures as a function of spatial frequency (Fig. 4B). A typical FSC curve has a value close to +1 at low frequencies (low-resolution details are highly correlated) and decays to zero at higher frequencies (structures differ in high-resolution details). The frequency at which the FSC curve drops below a certain threshold determines the final resolution of the structure. However, it must be noted that the resolution of a cryo-EM model is usually not uniform. It is typically highest in the well-ordered regions of the macromolecule (e.g., centres of globular domains, etc.), while some parts of the map can have significantly lower resolution due to the intrinsic flexibility of the molecule or for technical reasons (e.g., local reconstruction accuracy, non-isotropic distribution of viewing orientations, etc.) [64]. Several threshold values for assessing the resolution can be found in the literature (e.g., the 0.143 criterion that is believed to give resolution values comparable to those obtained in X-ray crystallography for a figure-of-merit (FOM) of 0.5 ; a more stringent criterion of 0.5 is also used). The "gold standard" introduced in 2012 requires that the FSC curve is calculated from two completely independent maps [65], but there is no single objective threshold value for assessing the resolution [11]. A similar problem exists in assessing the quality of the reconstructed 3D map. In SPR it is not possible to easily compare the final 3D model with the experimental data, as is typically done in X-ray crystallography, where the R-factor expresses the difference between the calculated and observed 3D structure factors. Calculated 3D EM maps could be in principle directly compared to the real space 2D projections recorded in TEM, but the single particle images are very noisy, which precludes analysis of the differences

\section{SUMMARY, FUTURE PERSPECTIVES, AND CURRENT LIMITATIONS}

Recent advances in the cryo-EM of biological macromolecules have led to the "resolution revolution" during the last few years. Thanks to the development of better imaging devices (i.e., DEDs), automation of the data collection process, and continual improvements in the available software, it is now possible to routinely obtain near-atomic-resolution 3D maps of intact biological macromolecules. One major resolution-limiting factor in cryo-EM is the inherent mobility of some large macromolecular complexes. Although mild chemical cross-linking can often be used to stabilize such molecules, it is possible that the mobile parts of many important biological macromolecules may never be visualized at high resolution.

Nevertheless, there is always room for improvement in cryo-EM methodology. One of the major challenges in cryoEM is to improve the contrast of low-dose images. Recently introduced phase plates can boost the contrast of TEM images and allow image collection without significant defocus [35]. This hardware upgrade, however, must be followed by a modification of the SPR algorithms that are presently optimized for processing of defocused images. Another unresolved problem is how to reduce the beam-induced movement of the specimen [34]. Recording images in movie-mode can partially compensate for this problem, but the development of better support materials can further improve TEM image quality [67]. All of these improvements, together with the continued development of electron optics, will expand the current resolution limits and enable the analysis of even smaller macromolecules.

A related technique that is ready for a similar "resolution revolution" is cryo-electron tomography with subtomo- 
gram averaging [46]. The current resolution limit (typically $2-4 \mathrm{~nm}$ ) is far from near-atomic, but the first structures below $10 \AA$ are already available [47]. This suggests the very tempting possibility of studying structural biology in situ and opens a new field of cellular structural biology.

\section{REFERENCES}

1. Rupp B (2009) Biomolecular crystallography: principles, practice, and application to structural biology. Garland Science

2. Berman HM, Westbrook J, Feng Z, Gilliland G, Bhat TN, Weissig H, Shindyalov IN, Bourne PE (2000) The Protein Data Bank. Nucleic Acids Res 28: 235-242

3. Wüthrich K (1986) NMR of Proteins and Nucleic Acids. Wiley-Interscience

4. Frank J (2006) Three-dimensional electron microscopy of macromolecular assemblies: visualization of biological molecules in their native state. Oxford University Press

5. Callaway E (2015) The revolution will not be crystallized: a new method sweeps through structural biology. Nature 525: 172-174

6. Wang RY, Kudryashev M, Li X, Egelman EH, Basler M, Cheng Y, Baker D, DiMaio F (2015) De novo protein structure determination from near-atomic-resolution cryo-EM maps. Nat Methods 12: 335-338

7. Uluç K, Kujoth GC, Başkaya MK (2009) Operating microscopes: past, present, and future. Neurosurg Focus 27: E4

8. Kruger DH, Schneck P, Gelderblom HR (2000) Helmut Ruska and the visualisation of viruses. Lancet 355: 1713-1717

9. De Carlo S, Harris JR (2011) Negative staining and cryo-negative staining of macromolecules and viruses for TEM. Micron 42: 117-131

10. Adrian M, Dubochet J, Lepault J, McDowall AW (1984) Cryo-electron microscopy of viruses. Nature 308: 32-36

11. Cheng Y, Grigorieff N, Penczek PA, Walz T (2015) A primer to single-particle cryo-electron microscopy. Cell 161: 438-449

12. Sachse C, Chen JZ, Coureux PD, Stroupe ME, Fändrich M, Grigorieff N (2007) High-resolution electron microscopy of helical specimens: a fresh look at Tobacco mosaic virus. J Mol Biol 371: 812-835

13. Zhang X, Settembre E, Xu C, Dormitzer PR, Bellamy R, Harrison SC, Grigorieff N (2008) Near-atomic resolution using electron cryomicroscopy and single-particle reconstruction. Proc Natl Acad Sci USA 105: 1867-1872

14. Tagari M, Newman R, Chagoyen M, Carazo JM, Henrick K (2002) New electron microscopy database and deposition system. Trends Biochem Sci 27: 589

15. Lawson CL, Baker ML, Best C, Bi C, Dougherty M, Feng P, van Ginkel G, Devkota B, Lagerstedt I, Ludtke SJ, Newman RH, Oldfield TJ, Rees I, Sahni G, Sala R, Velankar S, Warren J, Westbrook JD, Henrick K, Kleywegt GJ, Berman HM, Chiu W (2011) EMDataBank.org: unified data resource for CryoEM. Nucleic Acids Res 39: D456-D464

16. Campbell MG, Veesler D, Cheng A, Potter CS, Carragher B (2015) 2.8 A resolution reconstruction of the Thermoplasma acidophilum 20 S proteasome using cryo-electron microscopy. eLIFE 4: e06380

17. Banerjee S, Bartesaghi A, Merk A, Rao P, Bulfer SL, Yan Y, Green N, Mroczkowski B, Neitz RJ, Wipf P, Falconieri V, Deshaies RJ, Milne JL, Huryn D, Arkin M, Subramaniam S (2016) 2.3 Å resolution cryo-EM structure of human p97 and mechanism of allosteric inhibition. Science 351: 871-875

18. Voorhees RM, Fernández IS, Scheres SH, Hegde RS (2014) Structure of the mammalian ribosome-Sec61 complex to $3.4 \AA$ resolution. Cell 157: $1632-1643$

19. Fischer N, Neumann P, Konevega AL, Bock LV, Ficner R, Rodnina MV, Stark H (2015) Structure of the E. coli ribosome-EF-Tu complex at $<3$ Å resolution by $\mathrm{C}_{\mathrm{s}}$-corrected cryo-EM. Nature 520: 567-570

20. Bartesaghi A, Merk A, Banerjee S, Matthies D, Wu X, Milne JL, Subramaniam S (2015) $2.2 \AA$ resolution cryo-EM structure of $\beta$-galactosidase in complex with a cell-permeant inhibitor. Science 348: 1147-1151

21. Merk A, Bartesaghi A, Banerjee S, Falconieri V, Rao P, Davis MI, Pragani R, Boxer MB, Earl LA, Milne JL, Subramaniam S (2016) Breaking
cryo-EM resolution barriers to facilitate drug discovery. Cell 165: 1698 1707

22. Nguyen TH, Galej WP, Fica SM, Lin PC, Newman AJ, Nagai K (2016) CryoEM structures of two spliceosomal complexes: starter and dessert at the spliceosome feast. Curr Opin Struct Biol 36: 48-57

23. Chang L, Zhang Z, Yang J, McLaughlin SH, Barford D (2015) Atomic structure of the APC/C and its mechanism of protein ubiquitination. Nature 522: 450-454

24. Liao M, Cao E, Julius D, Cheng Y (2013) Structure of the TRPV1 ion channel determined by electron cryo-microscopy. Nature 504: 107-112

25. Huynh KW, Cohen MR, Jiang J, Samanta A, Lodowski DT, Zhou ZH, Moiseenkova-Bell VY (2016) Structure of the full-length TRPV2 channel by cryo-EM. Nat Commun 7: 11130

26. Bai XC, Yan C, Yang G, Lu P, Ma D, Sun L, Zhou R, Scheres SH, Shi Y (2015) An atomic structure of human $\gamma$-secretase. Nature 525: 212-217

27. Egerton R (2006) Physical principles of electron microscopy: an introduction to TEM, SEM, and AEM. Springer US

28. Jensen G (2013-2014) Getting started in cryo-EM. On-line lecture available at http:/ / cryo-em-course.caltech.edu

29. Faruqi AR, Henderson R (2007) Electronic detectors for electron microscopy. Curr Opin Struct Biol 17: 549-555

30. Fan GY, Ellisman MH (2000) Digital imaging in transmission electron microscopy. J Microsc 200: 1-13

31. Ruskin RS, Yu Z, Grigorieff N (2013) Quantitative characterization of electron detectors for transmission electron microscopy. J Struct Biol 184: 385-393

32. Cabra V, Samsó M (2015) Do's and Don'ts of Cryo-electron Microscopy: a primer on sample preparation and high quality data collection for macromolecular 3D reconstruction. J Vis Exp: 52311

33. Harris JR (2015) Transmission electron microscopy in molecular structural biology: A historical survey. Arch Biochem Biophys 581: 3-18

34. Henderson R (2015) Overview and future of single particle electron cryomicroscopy. Arch Biochem Biophys 581: 19-24

35. Schröder RR (2015) Advances in electron microscopy: A qualitative view of instrumentation development for macromolecular imaging and tomography. Arch Biochem Biophys 581: 25-38

36. Thompson RF, Walker M, Siebert CA, Muench SP, Ranson NA (2016) An introduction to sample preparation and imaging by cryo-electron microscopy for structural biology. Methods 100: 3-15

37. Grassucci RA, Taylor DJ, Frank J (2007) Preparation of macromolecular complexes for cryo-electron microscopy. Nat Protoc 2: 3239-3246

38. Kastner B, Fischer N, Golas MM, Sander B, Dube P, Boehringer D, Hartmuth K, Deckert J, Hauer F, Wolf E, Uchtenhagen H, Urlaub H, Herzog F, Peters JM, Poerschke D, Lührmann R, Stark H (2008) GraFix: sample preparation for single-particle electron cryomicroscopy. Nat Methods 5: 53-55

39. Ohi M, Li Y, Cheng Y, Walz T (2004) Negative staining and image classification - powerful tools in modern electron microscopy. Biol Proced Online 6: 23-34

40. Danev R, Baumeister W (2016) Cryo-EM single particle analysis with the Volta phase plate. eLIFE 5: e13046

41. Rochat RH, Hecksel CW, Chiu W (2014) Cryo-EM techniques to resolve the structure of HSV-1 capsid-associated components. Methods Mol Biol 1144: 265-281

42. Guo F, Jiang W (2014) Single particle cryo-electron microscopy and 3-D reconstruction of viruses. Methods Mol Biol 1117: 401-443

43. Rosenthal PB, Henderson R (2003) Optimal determination of particle orientation, absolute hand, and contrast loss in single-particle electron cryomicroscopy. J Mol Biol 333: 721-745

44. Henderson R, Chen S, Chen JZ, Grigorieff N, Passmore LA, Ciccarelli L, Rubinstein JL, Crowther RA, Stewart PL, Rosenthal PB (2011) Tiltpair analysis of images from a range of different specimens in single-particle electron cryomicroscopy. J Mol Biol 413: 1028-1046

45. Yahav T, Maimon T, Grossman E, Dahan I, Medalia O (2011) Cryo-electron tomography: gaining insight into cellular processes by structural approaches. Curr Opin Struct Biol 21: 670-677 
46. Briggs JA (2013) Structural biology in situ - the potential of subtomogram averaging. Curr Opin Struct Biol 23: 261-267

47. Schur FK, Hagen WJ, de Marco A, Briggs JA (2013) Determination of protein structure at $8.5 \AA$ resolution using cryo-electron tomography and sub-tomogram averaging. J Struct Biol 184: 394-400

48. Shaikh TR, Gao H, Baxter WT, Asturias FJ, Boisset N, Leith A, Frank J (2008) Spider image processing for single-particle reconstruction of biological macromolecules from electron micrographs. Nat Protoc 3: 1941-1974

49. Tang G, Peng L, Baldwin PR, Mann DS, Jiang W, Rees I, Ludtke SJ (2007) EMAN2: an extensible image processing suite for electron microscopy. J Struct Biol 157: 38-46

50. Scheres SH (2012) RELION: Implementation of a Bayesian approach to cryo-EM structure determination. J Struct Biol 180: 519-530

51. Lyumkis D, Brilot AF, Theobald DL, Grigorieff N (2013) Likelihood-based classification of cryo-EM images using FREALIGN. J Struct Biol 183: 377-388

52. Voss NR, Potter CS, Smith R, Carragher B (2010) Software tools for molecular microscopy: an open-text Wikibook. Methods Enzymol 482: 381-392

53. Zanetti G, Riches JD, Fuller SD, Briggs JA (2009) Contrast transfer function correction applied to cryo-electron tomography and sub-tomogram averaging. J Struct Biol 168: 305-312

54. Penczek PA (2010) Image restoration in cryo-electron microscopy. Methods Enzymol 482: 35-72

55. Zhu J, Penczek PA, Schröder R, Frank J (1997) Three-dimensional reconstruction with contrast transfer function correction from energy-filtered cryoelectron micrographs: procedure and application to the $70 \mathrm{~S}$ Escherichia coli ribosome. J Struct Biol 118: 197-219

56. Langlois R, Pallesen J, Ash JT, Nam Ho D, Rubinstein JL, Frank J (2014) Automated particle picking for low-contrast macromolecules in cryo-electron microscopy. J Struct Biol 186: 1-7

57. Scheres SH (2010) Classification of structural heterogeneity by maximum-likelihood methods. Methods Enzymol 482: 295-320
58. Leschziner A (2010) The orthogonal tilt reconstruction method. Methods Enzymol 482: 237-262

59. Penczek PA, Frank J, Spahn CM (2006) A method of focused classification, based on the bootstrap 3D variance analysis, and its application to EF-G-dependenttranslocation. J Struct Biol. 154: 184-194

60. Scheres SH, Gao H, Valle M, Herman GT, Eggermont PP, Frank J, Carazo JM (2007) Disentangling conformational states of macromolecules in 3D-EM through likelihoodoptimization. Nat Methods 4: 27-29

61. Plaschka C, Larivière L, Wenzeck L, Seizl M, Hemann M, Tegunov D, Petrotchenko EV, Borchers CH, Baumeister W, Herzog F, Villa E, Cramer P (2015) Architecture of the RNA polymerase II-Mediator core initiation complex. Nature 518: 376-380

62. Sigworth FJ, Doerschuk PC, Carazo JM, Scheres SH (2010) An introduction to maximum-likelihood methods in cryo-EM. Methods Enzymol 482: 263-294

63. van Heel M, Schatz M (2005) Fourier shell correlation threshold criteria. J Struct Biol 151: 250-262

64. Cardone G, Heymann JB, Steven AC (2013) One number does not fit all: mapping localvariations in resolution in cryo-EM reconstructions. J Struct Biol 184: 226-236

65. Henderson R, Sali A, Baker ML, Carragher B, Devkota B, Downing KH, Egelman EH, Feng Z, Frank J, Grigorieff N, Jiang W, Ludtke SJ, Medalia O, Penczek PA, Rosenthal PB, Rossmann MG, Schmid MF, Schröder GF, Steven AC, Stokes DL, Westbrook JD, Wriggers W, Yang H, Young J, Berman HM, Chiu W, Kleywegt GJ, Lawson CL (2012) Outcome of the first electron microscopy validation task force meeting. Structure 20: 205-214

66. Falkner B, Schröder GF (2013) Cross-validation in cryo-EM-based structural modeling. Proc Natl Acad Sci USA 110: 8930-8935

67. Russo CJ, Passmore LA (2016) Ultrastable gold substrates: properties of a support for high-resolution electron cryomicroscopy of biological specimens. J Struct Biol 193: 33-44

\title{
Wprowadzenie do wysokorozdzielczej kriogenicznej mikroskopii elektronowej Mariusz Czarnocki-Cieciura, Marcin Nowotny ${ }^{\bowtie}$
}

\author{
Laboratorium Struktury Białka, Międzynarodowy Instytut Biologii Molekularnej i Komórkowej w Warszawie, ul. Ks. Trojdena 4, 02-109 Warsza- \\ wa, Polska \\ e-mail: mnowotny@iimcb.gov.pl
}

Słowa kluczowe: mikroskopia elektronowa, cryo-EM, barwienie negatywowe, analiza pojedynczych cząsteczek, biologia strukturalna

\section{STRESZCZENIE}

Przez wiele lat biologia strukturalna zdominowana była przez dwie techniki, krystalografię rentgenowską oraz spektroskopię jądrowego rezonansu magnetycznego (NMR). Tradycyjna kriogeniczna mikroskopia elektronowa (cryo-EM) makrocząsteczek biologicznych pozwalała na uzyskiwanie struktur o rozdzielczościach, które nie przekraczały 6-10 A. Istotny rozwój transmisyjnych mikroskopów elektronowych, w tym w szczególności detektorów elektronów, który nastąpił w ostatnich latach, a także ciągłe udoskonalanie oprogramowania do analizy obrazów, doprowadziły do przełamania tego limitu rozdzielczości. Najnowsze struktury biologicznych makrocząsteczek uzyskiwane przy użyciu techniki cryo-EM charakteryzują się niemal atomową rozdzielczością $(<2.5 \AA ̊$ ). Masa cząsteczkowa najmniejszych z analizowanych makrocząsteczek nie przekracza $100 \mathrm{kDa}$, a wiele z nich nie posiada symetrii. Dzięki temu kriogeniczna mikroskopia elektronowa staje się obecnie preferowaną metodą analizy złożonych kompleksów białkowych, których wielkość oraz dynamika uniemożliwiają wykorzystanie innych metod biologii strukturalnej. 